\title{
Associação entre frequência de constipação e desfecho clínico em paciente crítico
}

\author{
Association between constipation frequency and clinical outcome in critically ill patients
}

\section{DOI: $10.37111 /$ braspenj.2019344011}

\section{Camila Melo de Araújo}

Edcleide Oliveira dos Santos Olinto ${ }^{2}$

Gina Araújo Martins Feitosa ${ }^{3}$

Izaura Odir Lima Gomes da Costa ${ }^{4}$

Janine Maciel Barbosa ${ }^{5}$

Ericka Vilar Bôtto Targino 6

Paloma Egídio Andrade de Sousa'

\section{Unitermos:}

Constipação intestinal. Unidades de terapia intensiva. Cuidados críticos.

\section{Keywords:}

Constipation. Intensive care units. Critical care.

\section{Endereço para correspondência:}

Camila Melo de Araújo

Hospital Universitário Lauro Wanderley (HULW/ UFPB/EBSERH)

Rua Tab. Stanislau Eloy, 585 - Castelo Branco - João

Pessoa, PB, Brasil - CEP 58050-585

E-mail: camilamelonutricao@gmail.com

\section{Submissão}

19 de julho de 2019

Aceito para publicação

8 de novembro de 2019

\begin{abstract}
RESUMO
Introdução: A constipação intestinal é uma complicação comum em pacientes críticos internados em unidade de terapia intensiva (UTI), devido a alguns fatores desencadeantes, como: limitação ao leito, uso de sedativos e opioides, drogas vasoativas, mediadores inflamatórios, entre outros, estando relacionada a um pior prognóstico. Método: Pesquisa quantitativa, descritiva, realizada em adultos, de ambos os sexos, admitidos na UTI de um hospital universitário, no período de março a dezembro de 2018. Foram coletadas das fichas de avaliação e acompanhamento nutricional, as variáveis gênero, idade (I), período de internamento na UTI, data de alta ou óbito. Foi avaliada a presença de constipação intestinal (ausência de evacuações > três dias). Após coleta, os dados foram tabulados em planilhas do software Microsoft Excel e, posteriormente, analisados pelo Statistical Package for the Social Sciences (SPSS) versão 13.0, sendo usado para diferença estatística, quando valor $p<0,05$, o teste Qui-Quadrado. Resultados: A amostra foi composta por 116 pacientes, sendo $46,6 \%$ homens e $53,4 \%$ mulheres, com idade média de 45 (IQ 31-53) anos. A ocorrência de constipação intestinal teve uma média de $47 \%$ (sendo a meta $<20 \%$ ). Em relação ao desfecho, o grupo de pacientes que apresentou constipação teve maior mortalidade $(27,5 \%$ versus $14,5 \%)$, porém não se confirmou estatisticamente $(p>0,05)$. A maioria dos pacientes apresentou risco nutricional baixo $(61,5 \%)$, no entanto, aqueles que apresentaram risco nutricional alto $(38,5 \%)$ houve uma tendência maior à constipação, mas essa relação não se confirmou estatisticamente $(p>0,05)$. Conclusão: Foi encontrada elevada frequência de constipação intestinal em pacientes graves. A constipação parece estar associada a pior prognóstico em pacientes críticos e é primordial que seja identificada e tratada. Diante desse resultado, faz-se necessária a criação de protocolos e planos de ação para prevenção e tratamento desse distúrbio nas unidades de terapia intensiva.
\end{abstract}

\section{ABSTRACT}

Introduction: Constipation is a common complication in critically ill patients hospitalized in an intensive care unit (ICU) due to some triggering factors such as: bed limitation, use of sedatives and opioids, vasoactive drugs, inflammatory mediators, etc. to a worse prognosis. Methods: A quantitative and descriptive study was conducted on adults of both genders hospitalized in the ICU of a university hospital from March to December 2018. Variables were collected from evaluation and nutritional monitoring forms, including: gender, age, length of ICU hospitalization, date of discharge or death. The presence of intestinal constipation (absence of bowel movements> three days) was evaluated. After collection, the data were tabulated in Microsoft Excel software spreadsheets and later analyzed by the Statistical Package for the Social Sciences (SPSS) version 13.0, and the chi-square test was used for statistical difference ( $p<0.05$ to accept the alternative hypothesis). Results: The sample consisted of 116 patients, $46.6 \%$ men and $53.4 \%$ women, with a mean age of 45 (IR 31-53) years. The occurrence of constipation averaged $47 \%$ (target $<20 \%$ ). Regarding the outcome, the group of patients with constipation had higher lethality $(27.5 \%$ versus $14.5 \%)$, but was not statistically confirmed ( $p>0.05)$. Most patients had low nutritional risk (61.5\%), however, those with high nutritional risk (38.5\%) had a greater tendency to constipation, but this relationship was not statistically confirmed ( $p>0.05$ ). Conclusion: A high frequency of constipation was found in critically ill patients. Constipation appears to be associated with a worse prognosis in critically ill patients and is essential to identify and treat. Given this result, it is necessary to create protocols and action plans for the prevention and treatment of this disorder in intensive care units.

1. Nutricionista Residente pelo Programa de Residência Multiprofissional em Saúde Hospitalar - Ênfase em Paciente Crítico no Hospital Universitário Lauro Wanderley (RIMUSH/HULW/UFPB/EBSERH), João Pessoa, PB, Brasil.

2. Especialista em Nutrição Clínica e Nutrição Parenteral e Enteral, Nutricionista assistencial e da Equipe Multiprofissional de Terapia Nutriciona (EMTN) do Hospital Universitário Lauro Wanderley (RIMUSH/HULW/UFPB/EBSERH), João Pessoa, PB, Brasil.

3. Especialista em Saúde Pública, Nutricionista assistencial e da Equipe Multiprofissional de Terapia Nutricional (EMTN) do Hospital Universitário Lauro Wanderley (RIMUSH/HULW/UFPB/EBSERH), João Pessoa, PB, Brasil.

4. Especialista em Terapia Intensiva e Nutrição Parenteral e Enteral, Médica Nutróloga, coordenadora da EMTN do Hospital Universitário Lauro Wanderley (RIMUSH/HULW/UFPB/EBSERH), João Pessoa, PB, Brasil.

5. Doutora em Nutrição, Nutricionista assistencial do Hospital Universitário Lauro Wanderley (RIMUSH/HULW/UFPB/EBSERH), João Pessoa, PB, Brasil.

6. Especialista em Terapia Intensiva e Nutrição Parenteral e Enteral, Enfermeira assistencial e enfermeira da EMTN do Hospital Universitário Lauro Wanderley (RIMUSH/HULW/UFPB/EBSERH), João Pessoa, PB, Brasil. 


\section{INTRODUÇÃO}

A maior parte de pacientes potencialmente graves encontra-se em unidades de terapia intensiva (UTI), local de suporte avançado de vida, conhecido por ser equipado com tecnologia e equipe multiprofissional treinada para cuidar de pacientes que se beneficiam de monitorização detalhada e tratamentos invasivos. As dietas comumente utilizadas em terapia intensiva são isentas de fibras, visto alto risco de isquemia intestinal ou dismotilidade grave ${ }^{1,2}$. Além disso, alterações ocorridas na fisiologia gastrointestinal do doente crítico estão relacionadas aos cuidados despendidos para tratar a doença ${ }^{3}$. A soma desses fatores termina por desencadear episódios de constipação.

A constipação é apontada na literatura como complicação gastrointestinal de grande incidência no doente crítico, que pode levar a quadro de evolução clínica desfavorável ${ }^{3}$. Esta complicação pode ser compreendida como um dos distúrbios da motilidade intestinal mais recorrente em pacientes em uso de nutrição enteral. Contudo, costuma ser pouco discutida quando comparada à diarreia, muitas vezes devido à falta de atenção da equipe multidisciplinar ${ }^{4}$.

Essa complicação gastrointestinal encontra-se associada a quadros como atraso no trânsito do intestino delgado, vômito, distensão abdominal, obstrução colônica, risco de perfuração intestinal, crescimento bacteriano, maior permanência em UTI e alimentação inadequada ${ }^{5}$. Além disso, pode ocorrer conjuntamente com gastroparesia, ileoparesia e, consequentemente, levar a atraso no suporte nutricional ${ }^{4}$. Portanto, verificar a influência da constipação sobre desfechos clínicos, tais como mortalidade, infecção bacteriana e maior tempo de ventilação mecânica, torna-se fundamental para que se possa ter melhor direcionamento nas condutas a serem tomadas ${ }^{3}$.

A nutrição enteral é um método indicado para alimentação do paciente crítico, pois resulta em trofismo intestinal, estimula o sistema imunológico, preserva a barreira intestinal, reduz translocação bacteriana, incidência de sepse e falência multissistêmica. Apesar de possuir poucos efeitos adversos, apresenta como principal fator limitante o aparecimento de complicações gastrointestinais ${ }^{1,2}$. $O$ tratamento adequado para este tipo de complicação ainda permanece incerto. Laxantes e enemas são os mais utilizados, porém com eficácia ainda inconsistente. Pode-se verificar a ocorrência de efeitos colaterais significativos do uso da lactulose e de enemas relatado em alguns estudos'.

Diante do exposto, o presente trabalho teve como objetivo avaliar a associação entre frequência de constipação e desfecho clínico em pacientes críticos internados em uma UTI de um hospital universitário.

\section{MÉTODO}

Trata-se de um estudo quantitativo e descritivo, realizado na UTI do Hospital Universitário Lauro Wanderley (HULW/ UFPB/EBSERH), em João Pessoa-PB, no período de março a dezembro de 2018. Foram incluídos no estudo todos os pacientes adultos entre 18 e 99 anos, de ambos os sexos, cujo prontuário possuísse o formulário de acompanhamento nutricional adotado pelo serviço de nutrição do hospital, devidamente preenchido, com tempo de permanência na UTI igual ou superior a 72 horas e que fizeram uso da TNE pelas vias nasogástrica, nasoenteral, orogástrica, gastrostomia ou jejunostomia. Foram excluídos aqueles que evolvíram para alta ou óbito dentro das primeiras 72 horas e os que não fizerem uso da terapia de nutrição enteral (TNE).

Na referida instituição, a TN é realizada diariamente e de forma ininterrupta, conforme quadro clínico e necessidades nutricionais de cada paciente. Todos os pacientes submetidos à TNE recebem fórmulas enterais industrializadas em sistema aberto (prontas para uso) ou sistema fechado, manipuladas em sondário conforme legislação específica, com administração contínua (24 horas) controlada por bomba de infusão ou gravitacional. Conforme protocolo do serviço, utiliza-se dieta enteral normocalórica e hiperproteica (isenta de lactose, sacarose e glúten) acrescida de módulos calóricos e/ou proteicos conforme necessidades estimadas. No caso de pacientes que possuem necessidades metabólicas específicas, utiliza-se dieta enteral adequada a sua condição clínica.

A coleta de dados iniciou-se no dia da admissão e o acompanhamento dos pacientes foi realizado até o momento de descontinuação da TNE, óbito ou alta da unidade. Para coleta de dados, utilizou-se formulário previamente estruturado utilizado na rotina diária do nutricionista, onde são registradas informações sobre dados de identificação (idade, gênero), dados clínicos (diagnóstico, motivo de internação, tempo de internação, desfecho), dados antropométricos [peso, altura, circunferência do braço (CB) e altura do joelho] e variáveis relacionadas à nutrição enteral (dieta, via de acesso, administração, complicações gastrointestinais), bem como indicadores de qualidade da TN (jejum inadequado, atendimento de meta de volume infundido versus prescrito, diarreia e constipação).

Os dados foram digitados no programa Excel para Windows ${ }^{\circledR}$. As análises foram realizadas no Programa SPSS versão 13.0. As variáveis contínuas foram testadas quanto à normalidade pelo teste de Kolmogorov Smirnov. Os dados foram descritos utilizando frequências, medidas de tendência central e de variação, para variáveis normais, média e desviopadrão (DP) e, não-normais, mediana e intervalo interquartílico (IQ). Na comparação entre as proporções utilizou-se o teste do qui quadrado de Pearson ou com correção de Yates para tabelas $2 \times 2$. O nível de significância adotado foi de $p<0,05$. 
O projeto foi aprovado pelo Comitê de Ética em Pesquisa em Seres Humanos do Hospital Universitário Lauro Wanderley (CEP-HULW), CAAE n 131924.19.0.0000.5183, de acordo com a Resolução n 196/96 do Conselho Nacional de Saúde.

\section{RESULTADOS}

A amostra foi composta por 116 pacientes, de ambos os sexos, sendo a maioria do sexo feminino $(53,4 \%)$, com idade média de 46 anos (IQ 31-53 anos) e faixa etária prevalente a de adulto de meia-idade (54,5\%; entre 40 e 60 anos). Obteve-se que a principal via de acesso para 0 suporte nutricional foi a via oral $(55,6 \%)$, seguida da enteral $(30,6 \%)$. Dentre os pacientes analisados, houve $79,1 \%$ de alta como desfecho clínico (Tabela 1).

A Tabela 1 também descreve variáveis relacionadas ao estado nutricional e risco nutricional dos pacientes críticos em TN analisados. Observou-se que houve uma frequência de

Tabela 1-Características demográficas, clínicas e nutricionais de pacientes em uso de terapia nutricional enteral em unidade de terapia intensiva de um hospital universitário, 2019.

\begin{tabular}{|c|c|}
\hline Variável & $\mathrm{n}(\%)$ \\
\hline \multicolumn{2}{|l|}{ Sexo } \\
\hline Masculino & $54(46,6)$ \\
\hline Feminino & $62(53,4)$ \\
\hline \multicolumn{2}{|l|}{ Idade } \\
\hline $18 \mid-40$ anos & $49(44,5)$ \\
\hline $40 \mid-60$ anos & $60(54,5)$ \\
\hline$\geq 60$ anos & $1(0,9)$ \\
\hline \multicolumn{2}{|l|}{ Via de acesso } \\
\hline Oral & $60(55,6)$ \\
\hline Enteral & $33(30,6)$ \\
\hline Parenteral & $2(1,9)$ \\
\hline Mista & $13(12,0)$ \\
\hline \multicolumn{2}{|l|}{ Desfecho } \\
\hline Alta & $91(79,1)$ \\
\hline Óbito & $24(20,9)$ \\
\hline \multicolumn{2}{|c|}{ Estado nutricional (IMC) } \\
\hline Desnutrido & $16(17,4)$ \\
\hline Eutrófico & $40(43,5)$ \\
\hline Sobrepeso/Obeso & $36(39,1)$ \\
\hline \multicolumn{2}{|c|}{ Estado nutricional (CB) } \\
\hline Desnutrido & $27(73,0)$ \\
\hline Eutrófico & $8(21,6)$ \\
\hline Sobrepeso/Obeso & $2(5,4)$ \\
\hline \multicolumn{2}{|l|}{ Nutric Escore } \\
\hline Baixo & $24(61,5)$ \\
\hline Alto & $15(38,5)$ \\
\hline
\end{tabular}

$\mathrm{CB}=$ circunferência do braço; IMC = índice de massa corporal. desnutrição quatro vezes maior pela CB quando comparado ao índice de massa corporal (IMC, 73\% versus 17,4\%). Em relação ao risco nutricional, obteve-se que quase um terço da amostra apresentou alto risco nutricional (38,5\%).

Dentre as principais intercorrências no trato gastrointestinal, observou-se que a diarreia esteve presente em 12,1\% dos analisados, enquanto a ocorrência de constipação intestinal foi de $47 \%$. Em relação ao tempo de duração, encontrou-se média de 3 dias $( \pm 1,8)$ para diarreia e 7 dias $( \pm 3,3)$ para constipação.

A Tabela 2 apresenta a associação da constipação com variáveis demográficas, clínicas e nutricionais. Em relação ao sexo e à idade, houve maior frequência nos indivíduos com menos de 40 anos de idade ( $52 \%$ versus $48 \%, p>0,05$ ), mas

Tabela 2 - Fatores associados à constipação em pacientes em uso de terapia nutricional enteral em unidade de terapia intensiva de um hospital universitário, 2019.

\section{Constipação}

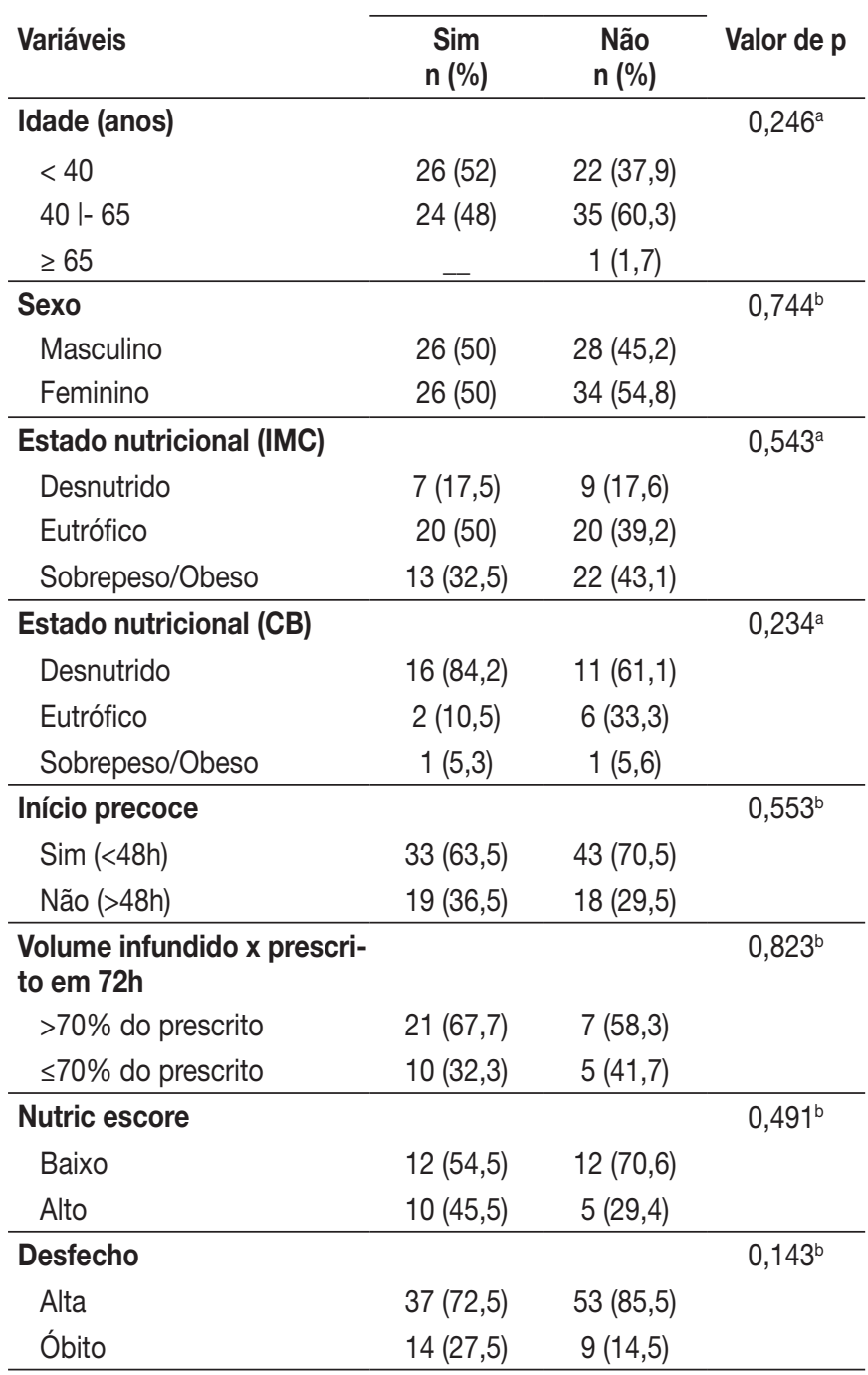

aTeste de Pearson; ' Correção de Yates.

$\mathrm{CB}$ = circunferência do braço; IMC = índice de massa corporal. 
frequência igual entre os sexos $(50 \%, p>0,05)$. Em relação às variáveis clínicas e nutricionais, os dados apontam que, entre aqueles que apresentaram constipação, houve maior frequência de desnutrição pela CB $(84,2 \%$ versus $61,1 \%)$, frequência de início tardio da TN (36,5\% versus $29,5 \%)$, risco nutricional pelo Nutric escore $(45,5 \%$ versus $29,4 \%)$ e óbito como desfecho clínico (27,5\% versus 14,5\%). Contudo, não houve diferença estatisticamente significante em nenhuma dessas análises $(p>0,05)$.

\section{DISCUSSÃO}

A análise dos resultados demonstrou que há uma incidência significativa de constipação. Considerando a definição como a ausência de evacuações por 3 dias consecutivos após o início da terapia nutricional, 47\% da amostra apresentaram constipação. Na literatura consultada, houve divergência em relação ao critério de definição da constipação, o que dificulta comparação com o presente estudo. Porém, alguns estudos com características semeIhantes à presente casuística também obtiveram frequência significante de constipação.

Batassini e Beghetto 6 , em estudo realizado em centro de cuidados intensivos composto por um total de 40 leitos clínicos e cirúrgicos em um hospital universitário brasileiro de alta complexidade, encontraram frequência superior de constipação (75,8\%). Prat et al. ${ }^{7}$, em estudo observacional prospectivo realizado em duas UTIs com 22 leitos no total, em dois hospitais universitários franceses, obtiveram frequência semelhante (51,9\%). Enquanto no estudo conduzido por Pérez-Sánchez et al. 8 , em UTI polivalente de hospital terciário espanhol, evidenciou prevalência de 63\%. Assim sendo, vários estudos realizados em UTIs confirmam que a constipação é uma complicação real em pacientes críticos e que o uso de um protocolo minimiza a incidência ${ }^{9-11}$.

Deve-se ressaltar que a frequência de constipação intestinal é utilizada como um indicador de qualidade de terapia nutricional e tem como meta a ser atingida um valor inferior a $20 \%{ }^{12}$. Contudo, na presente casuística, observou-se que essa meta não foi atingida em todo período analisado.

A análise revelou que não houve associação estatisticamente significante em relação ao gênero, à idade e à presença de constipação. Contudo, a associação entre sexo feminino e constipação intestinal, assim como o fato de que o idoso apresenta maior frequência desse distúrbio, é discutido na literatura ${ }^{13}$. Na análise das variáveis estado nutricional obtido pela CB e risco nutricional pelo Nutric escore, constatou-se maior frequência de constipação entre os desnutridos e os pacientes com alto risco nutricional. Em relação ao indicador IMC, observou-se maior frequência de constipação nos eutróficos (50\%), o que corrobora com o estudo de Klaus et al. ${ }^{14}(54,1 \%)$.
As complicações gastrointestinais relacionadas à TNE podem afetar negativamente o resultado clínico nutricional de pacientes hospitalizados. Diarreia e constipação são complicações frequentemente encontradas em pacientes em TNE, sendo distúrbios da motilidade intestinal associados a farmacoterapia, hidratação, estado nutricional e idade. Observando essas complicações no presente estudo, encontrou-se frequência de diarreia menor que a constipação $(12,1 \%$ versus $47 \%)$. Bittencourt et al. ${ }^{4}$, em estudo conduzido com 110 adultos internados em Hospital de São Paulo (SP-Brasil) alimentados exclusivamente por nutrição enteral através de sonda, obtiveram achados semelhantes, com frequência de constipação de $70 \%$ e diarreia de $13 \%$. Achados adicionais desses autores apontam para uma relação significativa entre o uso de fórmula enteral sem fibra e constipação.

A constipação, bem como suas implicações, parece receber pouca atenção, uma vez que exige menos atenção da equipe multidisciplinar. Entretanto, esse distúrbio pode estar presente concomitantemente a outros, como gastroparesia e ileoparesia. Estas complicações podem levar a atraso no suporte nutricional e a ingestão inadequada de nutrientes, interferindo, consequentemente, no prognóstico dos pacientes ${ }^{15}$.

Em relação à associação entre início precoce do suporte nutricional enteral e frequência de constipação, não foram encontradas associações significantes. Essa análise tinha como objetivo avaliar se o início precoce da terapia nutricional era capaz de influenciar a ocorrência de complicações gastrointestinais, incluindo a constipação. Nassar et al. ${ }^{15}$ encontraram que o início precoce esteve associado de maneira significante à menor frequência de constipação, mesmo tendo estabelecido como precoce o suporte iniciado em até 24 horas da admissão. No presente estudo, foi estabelecido como precoce a introdução em até 48 horas da admissão, conforme preconizado pela ASPEN ${ }^{16}$.

Quanto ao atendimento de meta de volume prescrito versus infundido, avaliado em $72 \mathrm{~h}$ após o início da terapia nutricional, não se encontrou diferença estatisticamente significante, demonstrando que a constipação intestinal não interferiu no alcance de metas nutricionais. Pacientes que evoluem com constipação frequentemente cursam com gastroparesia e ileoparesia, condições que atrasam o início e dificultam a progressão do suporte nutricional. Estes pacientes podem atrasar ou mesmo não atingir sua meta nutricional estimada pela via enteral ${ }^{17}$.

Um dos principais achados da presente casuística foi a tendência de maior frequência de desfecho negativo (óbito) entre os pacientes com constipação, porém não se confirmou estatisticamente. Gacouin et al. ${ }^{18}$, em estudo de coorte em uma UTI mista de 21 leitos de um hospital francês, demonstraram que as taxas de mortalidade foram 
significativamente maiores em pacientes com defecação tardia do que em pacientes com defecação precoce $(30 \%$ versus 18\%). A literatura diante de achados semelhantes aponta que a constipação intestinal pode estar ligada ao prognóstico de pacientes graves e levanta uma questão: constipação intestinal seria apenas um marcador de gravidade ou a expressão clínica de uma disfunção orgânica que necessita ser diagnosticada e tratada, a fim de modificar o prognóstico destes pacientes? ${ }^{17}$

O estudo tem algumas limitações, pois foi realizado em uma única UTI, assim os resultados podem não ser aplicáveis a UTIs de outras instituições. Um outro fator limitante foi o desconhecimento do padrão de evacuação anterior ao internamento de cada paciente. E, por último, a dificuldade de se obter registro fidedigno das intercorrências digestivas nos balanços de enfermagem na prática clínica.

\section{CONCLUSÃO}

A frequência de constipação intestinal em adultos críticos é elevada, sendo um assunto relevante dentro da terapia intensiva, necessitando de mais investigação para que haja uma padronização tanto para definição quanto para utilização de protocolos. Dessa forma, pode-se obter uma maior atenção da equipe multidisciplinar, chegar a soluções de maneira precoce, com vistas a obter melhor prognóstico clínico, atuando em diversos fatores que podem influenciar a constipação e os desfechos apresentados.

\section{REFERÊNCIAS}

1. López-Herce J. Gastrointestinal complications in critically ill patients: what differs between adults and children? Curr Opin Clin Nutr Metab Care. 2009;12(2):180-5.

2. Guerra TLS, Mendonça SS, Marshall NG. Incidência de constipação intestinal em uma unidade de terapia intensiva. Rev Bras Ter Intensiva. 2013;25(2):87-92.

3. Batassini E. Incidência e fatores associados à constipação: coorte prospectiva de pacientes adultos críticos [Dissertação de Mestrado]. Porto Alegre: Universidade Federal do Rio Grande do Sul, Escola de Enfermagem, Programa de Pós-graduação em Enfermagem; 2017.

4. Bittencourt AF, Martins JR, Logullo L, Shiroma G, Horie L, Ortolani MC, et al. Constipation is more frequent than diarrhea in patients fed exclusively by enteral nutrition: results of an observational study. Nutr Clin Pract. 2012;27(4):533-9.

5. Btaiche IF, Chan LN, Pleva M, Kraft MD. Critical illness, gastrointestinal complications, and medication therapy during enteral feeding in critically ill adult patients. Nutr Clin Pract. 2010;25(1):32-49.

6. Batassini É, Beghetto MG. Estreñimiento en una cohorte prospectiva de adultos críticos: porcentaje y motivo de su incidencia. Enferm Intensiva. 2019;30(3):127-34

7. Prat D, Messika J, Avenel A, Jacobs F, Fichet J, Lemeur M, et al. Constipation incidence and impact in medical critical care patients: importance of the definition criterion. Eur J Gastroenterol Hepatol. 2016;28(3):290-6.

8. Pérez-Sánchez J, Fernández-Boronat J, Martínez-Méndez E, Marín-Cagigas ML, Mota-Puerto D, Pérez-Román MC, et al. Evaluación y abordaje del estreñimiento en el paciente crítico. Enferm Intensiva. 2017;28(4):160-8.

9. Martínez MA, Gómez MJ, Pastor A. Prevención y tratamiento del estreñimiento en pacientes sometidos a cirugía cardíaca: estudio comparativo. Enferm Clin. 1999;9(3):105-8.

10. Rosón Ferreiro MJ, Fuentes LMR, Otero Pérez R, Sánchez Gómez MJ. Estreñimiento se opone a bienestar. Rev ROL Enf. 2004;27(5):33-8.

11. Lerga MC, López MP, Marraco M, Rioseco MJ, Margall MA. Valoración del patrón intestinal en pacientes de UCI mediante los registros de enfermería. Enferm Intensiva. 1994;5:1217.

12. Força-Tarefa de Nutrição Clínica, Força-Tarefa de Nutrição da Criança. Indicadores de qualidade em terapia nutricional: 10 anos de IQTN no Brasil: resultados, desafios e propostas. $3^{\mathrm{a}}$ ed. São Paulo: ILSI Brasil; 2018.

13. Garcia LB, Berolini S, Souza MV, Santos MSF, Pereira COM. Constipação intestinal: aspectos epidemiológicos e clínicos. Rev Saúde Pesqu. 2016;9(1):153-62.

14. Klaus JH, De Nardin V, Paludo J, Scherer F, Dal Bosco SM. Prevalência e fatores associados à constipação intestinal em idosos residentes em instituições de longa permanência. Rev Bras Geriatr Gerontol. 2015;18(4):835-43.

15. Nassar AP Jr, Silva FM, Cleva R. Constipation in intensive care unit: incidence and risk factors. J Crit Care. 2009;24(4):630.e9-12

16. McClave SA, Taylor BE, Martindale RG, Warren MM, Johnson DR, Braunschweig C, et al.; the A.S.P.E.N. Board of Directors and the American College of Critical Care Medicine. Guidelines for the provision and assessment of nutrition support therapy in the adult critically ill patient: Society of Critical Care Medicine (SCCM) and American Society for Parenteral and Enteral Nutrition (A.S.P.E.N.). JPEN J Parenter Enteral Nutr. 2016;40(2):159-211.

17. Azevedo RP, Freitas FGR, Ferreira EM, Machado FR. Constipação intestinal em terapia intensiva. Rev Bras Ter Intensiva. 2009;21(3):324-31.

18. Gacouin A, Camus C, Gros A, Isslame S, Marque S, Lavoué S, et al. Constipation in long-term ventilated patients: associated factors and impact on intensive care unit outcomes. Crit Care Med. 2010;38(10):1933-8.

Local de realização do estudo: Hospital Universitário Lauro Wanderley (RIMUSH/HULW/UFPB/EBSERH), João Pessoa, PB, Brasil.

Conflito de interesse: As autoras declaram não haver.

Foi apresentado como trabalho de destaque no XXIII Congresso Brasileiro de Nutrição Parenteral e Enteral, entre 20 a 23 de outubro de 2019, em Foz do Iguaçu-PR. 